\title{
On-chip DNA analysis of Tuberculosis based on magnetic nanoparticle clustering induced by rolling circle amplification products
}

Minero, Gabriel Antonio; Tefiku, Elisabeta; Garbarino, Francesca; Fock, Jeppe; Hansen, Mikkel Fougt

Published in:

I E E E Magnetics Letters

Link to article, DOI:

10.1109/Imag.2019.2959545

Publication date:

2020

Document Version

Peer reviewed version

Link back to DTU Orbit

Citation $(A P A)$ :

Minero, G. A., Tefiku, E., Garbarino, F., Fock, J., \& Hansen, M. F. (2020). On-chip DNA analysis of Tuberculosis based on magnetic nanoparticle clustering induced by rolling circle amplification products. I E E E Magnetics Letters, 11, [3100105]. https://doi.org/10.1109//mag.2019.2959545

\section{General rights}

Copyright and moral rights for the publications made accessible in the public portal are retained by the authors and/or other copyright owners and it is a condition of accessing publications that users recognise and abide by the legal requirements associated with these rights.

- Users may download and print one copy of any publication from the public portal for the purpose of private study or research.

- You may not further distribute the material or use it for any profit-making activity or commercial gain

- You may freely distribute the URL identifying the publication in the public portal 


\title{
On-chip DNA analysis of Tuberculosis based on magnetic nanoparticle clustering induced by rolling circle amplification products
}

\author{
Gabriel Antonio S. Minero ${ }^{1}$, Elisabeta Tefiku ${ }^{1}$, Francesca Garbarino ${ }^{1}$, Jeppe Fock ${ }^{2}$, and Mikkel F. \\ Hansen ${ }^{1}$ \\ ${ }^{1}$ Department of Health Technology, Building 345C, Technical University of Denmark, DK-2800, Kongens Lyngby, Denmark \\ ${ }^{2}$ BluSense Diagnostics, Fruebjergvej 3, DK-2100 Copenhagen, Denmark
}

Received 1 Apr 2016, revised 15 Apr 2016, accepted 20 Apr 2016, published 1 Jun 2016, current version 15 Jun 2016. (Dates will be inserted by IEEE; "published" is the date the accepted preprint is posted on IEEE Xplore ${ }^{\circledR}$; "current version" is the date the typeset version is posted on Xplore ${ }^{\circledR}$ ).

\begin{abstract}
We present on-chip implementation of an isothermal nucleic acid amplification assay with optomagnetic detection. Isothermal rolling circle amplification (RCA) was designed for the highly specific detection of a single point mutation in the sub-sequence of the katG catalase peroxidase gene responsible for resistance against the antibiotic isoniazid in Mycobacterium tuberculosis. The assay was integrated using streptavidin coated magnetic microbeads (MMBs) as a movable substrate to transport the synthetic katG target and products between the sequential processing steps. RCA products were detected via optomagnetic measurements of the binding of functionalized magnetic nanoparticles (MNPs). Binding of MNPs to RCA products lead to an increase of their hydrodynamic size and consequently a shift of the optomagnetic signal to lower frequencies. We first analyzed the affinity of DNA probes used for target capture on MMBs and binding of MNPs to RCA products, respectively. This enabled us to choose conditions where amplification products were released from the MMBs and their binding to MNPs was optimal. The optimized assay was transferred to a polymer chip with three connected chambers filled with reaction buffers for capture, RCA and detection. The chip was operated in a setup integrating temperature control, transport of the DNA on magnetic carriers and dose-dependent detection of MNP clustering. For a total assay time of about $2 \mathrm{~h}$, we demonstrated specific detection of the antibiotic resistance in synthetic Tuberculosis DNA target with a limit of detection of $4 \mathrm{pM}$.
\end{abstract}

Index Terms— Nucleic Acid Analysis, Lab-on-Chip, Biosensing, Magnetorelaxometry.

\section{INTRODUCTION}

Tuberculosis remains a major health concern with claimed 10.0 million people infected by Mycobacterium tuberculosis $(M T b)$ in 2017 [WHO 2017]. Routine clinical tests for MTb are culture based and require at least four weeks as well as a thorough quality assessment due to a large variation in time to positive result [Rageade 2014]. Additional 4-6 weeks are needed to screen for the response of $M T b$ to antibiotics. A faster detection of $M T b$ can help to reduce the spreading and high mortality of tuberculosis in the world.

It has been shown that certain mutations in chromosomal DNA of $M T b$ are responsible for the acquired antibiotic resistance towards the first-line drug, isoniazid [Domínguez 2016]. Such mutations can be recognized with very high specificity using padlock probe (PLP) ligation [Nilsson 1994]. PLP ligation is based on target-directed joining of the 5'- and 3'-terminals of a circular PLP and has been shown to discriminate a single base mutation in $M T b$ [Pavankumar 2016]. A joined PLP acts as template for rolling circle amplification (RCA), which forms a long single-stranded DNA concatemer. After $1 \mathrm{~h}$ of amplification, such a rolling circle product ( $\mathrm{RCP})$ contains

corresponding author: M. F. Hansen (mfha@dtu.dk). IEEE Magnetics Letters discourages courtesy authorship; please use the Acknowledgment section to thank your colleagues for routine contributions.

Digital Object Identifier: 10.1109/LMAG.XXXX.XXXXXXX (inserted by IEEE). about 1000 copies of the DNA sequence complementary to the PLP [Ali 2014].

The RCPs can be detected using fluorescence probes [Nilsson 2002], electroactive molecules [Zhang 2009], gold nanoparticles [Li 2010], magnetic microparticles [Lin 2013], magnetic nanoparticles [Mezger 2015] as well as other techniques [Pashchenko 2018, Zhou 2017]. Using an optomagnetic (OM) readout, we have previously demonstrated that binding of functionalized magnetic nanoparticles (MNPs) to RCPs resulted in a significant increase of the hydrodynamic size of the MNPs with a $200 \mathrm{fM}$ limit of detection (LOD) after $45 \mathrm{~min}$ of RCA [Minero 2019]. The OM readout is homogeneous and insensitive to sample matrix impurities or variations.

Handling of a target sample using magnetic microbeads (MMBs) in microfluidic devices is appealing as it enables target enrichment and multi-step RCA assay integration and on-chip automation [Sato 2010, Lu 2018, Hernandez-Neuta 2018, Garbarino 2019]. Controllable release of RCPs from MMB carriers can improve the performance of such a heterogeneous MMB-based assay [Minero 2018]. In our previous work, we demonstrated an integrated and automated RCA assay with no enrichment step or need for extra supply of liquids and a limit of detection of $2 \mathrm{pM}$ of a synthetic influenza DNA target [Garbarino 2019].

Herein, we present the tailoring and optimization of the assay for the highly specific detection of a synthetic DNA sub-sequence of the 
katG catalase peroxidase gene with the single base mutation responsible for the antibiotic resistance of $M T b$. The multi-step RCA assay comprises (0) DNA ligation, (1) DNA capture on MMBs, (2) RCA performed on MMBs, and (3) on-chip OM detection of the RCPs. The temperature of the OM detection is optimized to release RCPs from MMBs without compromising their binding to functionalized MNPs. Finally, we demonstrate feasibility of assay automation by implementing steps (1)-(3) on a multi-chamber chip in a setup integrating temperature control, magnetic transportation and $\mathrm{OM}$ detection.

\section{Methods}

\section{Fig. 1 here}

\section{A. Concept of integrated RCA assay}

Fig. 1 shows the steps of the molecular assay. In case of a match between the MTb DNA target and the PLP, the ligation step forms a circular DNA template for RCA. Subsequently, the DNA target with ligated PLP is captured on MMBs functionalized with a capture oligo (CO) and used as a primer for polymerization and RCA. The resulting single stranded RCPs contain several hundreds of binding sites for a detection oligo (DO) grafted onto MNPs. Finally, the binding of MNPs to the RCPs is detected using the OM technique.

To achieve an efficient diffusion of DO-MNPs to RCPs, it is essential that the RCPs are released from the MMBs that after onchip magnetic handling are confined at the bottom of the chip. Therefore, the $\mathrm{CO}$ and DO probes should be designed to control melting of the CO-target and DO-RCP hybrids at distinctly different temperatures such that RCP can be released on demand while still having efficient binding of the DO-MNPs to the RCPs.

\section{B. Experimental procedures}

We studied two synthetic DNA targets of $M T b$ with a single base difference, the ACC-katG (Mut) and the AGC-katG (WT), and implemented a Mut-specific PLP. The DNA targets and PLP sequences (Table S1) were adapted from Pavankumar et al. [2016].

For the DNA target capture, we used $0.2 \mathrm{mg} / \mathrm{mL}$ of $1 \mu \mathrm{m}$ diameter MyOne streptavidin C1 MMBs (ThermoFisher) functionalized with 3'-biotinylated $\mathrm{CO}$ probes (Table S1).

For the OM detection, we used multicore MNPs with a nominal diameter of $100 \mathrm{~nm}$ and a streptavidin coating (BNF starch, Micromod $\mathrm{GmbH}$ ) functionalized with 3'-biotinylated DO probes (Table S1). Previously, Fock et al. [2017] established that these MNPs have a remanent magnetic moment linked to anisotropic optical extinction properties. The MNP concentration in suspension was $0.05 \mathrm{mg} / \mathrm{mL}$.

First, off-chip assay processing combined with OM detection in 1chamber chips, as described by Minero et al. [2019], was used to: (1) characterize the temperature-induced denaturing (melting) of the CO-target hybrid and of hybrids formed between DO-MNPs; (2) optimize assay conditions for MMB sample handling and to benchmark results against the integrated assay.

Next, we integrated the assay on a lab-chip microfluidic platform containing three chambers interconnected via microchannels (Fig. 1) [Garbarino 2019]. Details on reagents and experimental protocols were as described by Garbarino et al. [2019], except for the reaction temperatures: After off-chip ligation $\left(60^{\circ} \mathrm{C}, 20 \mathrm{~min}\right), M T b$ targetPLP hybrids were captured on the MMBs in chamber $\mathrm{c} 1$ at $60^{\circ} \mathrm{C}$ for 2 min followed by cooling to $30^{\circ} \mathrm{C}$ for $5 \mathrm{~min}$ and additional $10 \mathrm{~min}$ incubation at $30^{\circ} \mathrm{C}$. RCA was performed by phi29 polymerase in chamber c2 at $38^{\circ} \mathrm{C}$ for $45 \mathrm{~min}$. During both capture and RCA, a motorized permanent magnet underneath the chip was moved back and forth to mix the MMBs and to boost diffusion of the reagents to MMBs. Finally, the MMBs with RCPs were transported to the detection chamber (c3), the magnet was moved away, the temperature was increased to $62.5^{\circ} \mathrm{C}$ and the binding of MNPs to the RCPs was monitored using OM measurements.

\section{Optomagnetic measurements}

The OM technique measures the modulation of the intensity of light transmitted through the MNP suspension in response to an oscillating magnetic field, $B(t)=B_{0} \sin (2 \pi f t)$, applied along the light path as function of the frequency, $f$. MNPs of a single size with linked optical and magnetic anisotropies give rise to a distinct peak in the $V_{2}^{\prime} / V_{0}$ vs. $f$ signal, where $V_{2}^{\prime}$ denotes the amplitude of the $\sin (4 \pi f t)$ component of the photodetector signal and $V_{0}$ is the average photodetector signal [Fock 2017]. The peak is located at a frequency, which is inversely related to the hydrodynamic size of the MNP [Fock 2017, Fock 2018]. When MNPs bind to RCPs, their hydrodynamic size is substantially increased. Therefore, the fraction of bound MNPs and thereby the concentration of a target can be quantified by the 'turn-off' of the $V_{2}^{\prime} / V_{0}$ signal from free MNPs at high frequencies. In this work, each OM spectrum was recorded in oscillating magnetic field with $B_{0}=1 \mathrm{mT}$ at 41 frequencies $f$ between $1 \mathrm{~Hz}$ and $2800 \mathrm{~Hz}$ in about $45 \mathrm{~s}$ using light from a light emitting diode with $\lambda=470 \mathrm{~nm}$.

\section{RESULTS \& DISCUSSION}

\section{A. Analysis of DNA probes}

To facilitate detection of RCPs, we developed a strategy to release RCPs from the MMBs without compromising their simultaneous binding to MNPs. We first characterized the stability of the COtarget and MNP-DO RCP hybrids, respectively, using a DNA melting assay [Minero 2017, Minero 2019]. In this assay, MNPs were functionalized either with $\mathrm{CO}$ probes (mimicking CO-MMBs) and RCPs were grown on them or they were functionalized with DO probes and mixed with ready-made RCPs. The stability of the obtained CO-target and DO-RCP hybrids was determined from OM measurements of the MNP-RCP OM spectra vs. temperature (Fig. 2) such that denaturation of the hybrids linking MNPs to the RCPs resulted in a decrease of the detected MNP size.

\section{Fig. 2. here}

For RCPs grown on targets attached to MNPs via hybridization to the CO probe (Fig. 2a), low-temperature OM spectra showed a peak at a very low frequency $(<1 \mathrm{~Hz})$. In contrast, low-temperature OM spectra of DO-MNPs bound to ready-made RCPs presented a positive peak at $f<1 \mathrm{~Hz}$ (Fig. 2b). This difference is due to the fact that RCPs have many sites complementary to DOs whereby several DO-MNPs can bind to each RCP. In contrast, only one CO-MNP 
can bind to each RCP. Clusters of DO-MNPs interact with light differently from single CO-MNPs [Fock 2017]. Upon increasing temperature, the $\mathrm{CO}$ as well as DO bridges to RCPs melt resulting in an increasing population of free MNPs detected at high frequencies.

Figure 2c shows the average OM signal at high frequencies (40$1000 \mathrm{~Hz}),\left\langle V_{2}^{\prime} / V_{0}\right\rangle$, vs. temperature for the spectra plotted in Fig. 2a and $2 \mathrm{~b}$. The results suggest that the CO-target hybrids have a broad melting transition at $45^{\circ} \mathrm{C}<T<75^{\circ} \mathrm{C}$, whereas the DO-RCP hybrids have a sharper melting behavior at $65^{\circ} \mathrm{C}<T<75^{\circ} \mathrm{C}$. The sharper melting behavior can be due to cooperative DO-RCP binding as each MNP can form many bonds to an RCP. These observations suggest that an optimal detection temperature is $50^{\circ} \mathrm{C}<T<65^{\circ} \mathrm{C}$.

\section{B. Temperature optimization for OM detection of RCA with discrimination of a single base mutation in MTb}

Fig. 3 here

Fig. 3 shows real-time on-chip OM detection of RCPs synthesized in tubes from $60 \mathrm{pM}$ target $\left(45 \mathrm{~min} \mathrm{RCA}\right.$ at $\left.38^{\circ} \mathrm{C}\right)$ at (a) $50^{\circ} \mathrm{C}$ and (b) $65^{\circ} \mathrm{C}$, respectively. The obtained $\mathrm{OM}$ spectra revealed depletion of free MNPs (at $40 \mathrm{~Hz}<f<1000 \mathrm{~Hz}$ ) and accumulation of MNP clusters (at $1 \mathrm{~Hz}<f<40 \mathrm{~Hz}$ ). The presence of the clusters in suspension was found in good agreement with melting of the COtarget bridges at $T \geq 50^{\circ} \mathrm{C}$. For assay quantification, we plotted the time-dependent fraction of MNPs bound to RCPs, $B_{\mathrm{MNP}}$, defined as $B_{\mathrm{MNP}}=1-\left\langle V_{2}^{\prime} / V_{0}\right\rangle(t) /\left\langle V_{2}^{\prime} / V_{0}\right\rangle\left(t_{0}\right)$ (Fig. 3c). The results show that the assay has very high specificity towards one base mutation as the signal obtained from the mismatched DNA target/PLP hybrid was indistinguishable from a no-template control (NTC). Temperatures above $60^{\circ} \mathrm{C}$ were found to give the highest $B_{\mathrm{MNP}}$ values after $30 \mathrm{~min}$ (Fig. 3d). Heating above $65^{\circ} \mathrm{C}$ was counterproductive as DO-RCP bridges would melt (Fig. $2 \mathrm{~b}$ ). Therefore, we chose $62.5^{\circ} \mathrm{C}$ as the detection temperature in the integrated on-chip assay.

\section{Dose-response analysis of MTb in integrated RCA assay}

\section{Fig. 4 here}

We performed dose-response analysis of the Mut Target - Mut PLP hybrids for different target concentrations. The PLPs (2 nM) were ligated off-chip. The ligated templates were mixed with $\mathrm{CO}-$ MMBs to obtain target DNA concentrations between $2 \mathrm{pM}$ and 100 pM. This was followed by magnetic separation of the MMBs and RCA. After $45 \mathrm{~min}$ of RCA, the reaction was terminated by magnetic separation of MMBs and followed by OM detection (Fig. 4). We compared results obtained with all reaction steps performed off-chip in tubes in a thermoshaker and the optomagnetic detection performed in a 1-chamber chip (Fig. 4a) with those obtained following the integrated strategy of Fig. 1, where all steps except for the ligation were integrated in the 3-chamber chip (Fig. 4b). We note a strong similarity of the obtained analyses such as the linear trend in the concentration range of $2-40 \mathrm{pM}$ with signal saturation above $60 \mathrm{pM}$. The obtained LODs of the RCPs synthesized off-chip (Fig. 4a) and on-chip (Fig. 4b) of $6 \mathrm{pM}$ and $4 \mathrm{pM}$, respectively, were similar.
As the detection took place under nominally identical conditions, the above observations suggest that the RCA efficiency was not compromised in the 3-chamber chip. However, in the integrated and automated OM-RCA format we note larger standard deviations as well as a larger signal cutoff value suggesting a lower reproducibility. The observed LODs compare well to previous results obtained for synthetic Influenza target [Minero 2019, Garbarino 2019] as well as to other works implementing magnetic carriers [Hernandez-Neuta 2018] and the OM detection [Strömberg 2008, Donolato 2015]. In this work, the existing assay protocol was tailored for detection of a single base mutation in synthetic $M T b$ target. In addition to assay integration, the MMB handling presented several advantages such as the separation of the target and RCPs from the excess of PLPs, which could otherwise compete with the DOs on the binding to RCPs. The LOD can be further lowered via implementation of an enrichment step using the CO-MMBs as a 'fishhook' to capture target DNA in a larger sample volume [Lu 2018].

One of the challenges of the RCA implementation on MMBs is the heterogeneity of the assay, which can lead to a substantial variation in the obtained readout signal. We addressed this heterogeneity using automated magnet-assisted mixing of MMBs as well as a controlled release of the synthesized RCPs from MMBs in the detection stage.

\section{CONCLUSION}

We optimized the on-chip ligation, amplification and optomagnetic detection of a synthetic DNA target of the katG peroxidase gene in $M T b$ and demonstrated detection of a matching Mut target down to $4 \mathrm{pM}$ whereas a mismatching WT target gave a signal identical to the negative control.

\section{ACKNOWLEDGMENT}

The work was supported by DFF project (\#4184-00121B). ET thanks Erasmus+ Program (Key action 1 A. Y. 2018-19) and Roberto Raiteri for sponsoring of the Erasmus program. JF acknowledges MUDP for support (MST-141-01415).

\section{REFERENCES}

Ali M M, Li F, Zhang Z, Zhang K, Kang D K, Ankrum J A, Le X C, Zhao W (2014), "Rolling circle amplification: a versatile tool for chemical biology, materials science and medicine," Chem. Soc. Rev., vol. 43, pp. 3324-3341.

Dominguez J, Cirillo D, Cobelens F, Eisenach K D, Gagneux S, Hillemann D, Horsburgh R, Molina-Moya B, Niemann S, Tortoli E, Whitelaw A, Lange C (2016) "Clinical implications of molecular drug resistance testing for Mycobacterium tuberculosis: A TBNET/RESIST-TB consensus statement," International Journal of Tuberculosis and Lung Disease, vol. 20, pp. 24-42.

Fock J, Jonasson C, Johansson C, Hansen M.F (2017) "Characterization of fine particles using optomagnetic measurements," Phys. Chem. Chem. Phys., vol. 19, pp. 8802-8814.

Garbarino F, Minero G A S, Rizzi G, Fock J, Hansen M F (2019) "Integration of rolling circle amplification and optomagnetic detection on a polymer chip," Biosens. Bioelectron., vol. 142, pp. 111485.

Hernández-Neuta I, Pereiro I, Ahlford A, Ferraro D, Zhang Q, Viovy J L, Descroix S, Nilsson M (2018) "Microfluidic magnetic fluidized bed for DNA analysis in continuous flow mode," Biosens. Bioelectron., 
vol. 102 , no. November 2017, pp. 531-539.

Li J, Deng T, Chu X, Yang R, Jiang J, Shen G, Yu R (2010) "Rolling circle amplification combined with gold nanoparticle aggregates for highly sensitive identification of single-nucleotide polymorphisms," Anal. Chem., vol. 82, pp. 2811-16.

Lin C, Zhang Y, Zhou X, Yao B, Fang Q (2013) "Naked-eye detection of nucleic acids through rolling circle amplification and magnetic particle mediated aggregation," Biosens. Bioelectron., vol. 47, pp. 515-519.

Lu W, Wang Y, Song S, Chen C, Yao B, Wang M (2018) "A fishhook probe-based rolling circle amplification assay for efficient isolation and detection of microRNA without total RNA extraction," Analyst, vol. 143, pp. 5046-5053.

Mezger A, Fock J, Antunes P, Østerberg F W, Boisen A, Nilsson M, Hansen M F, Ahlford A, Donolato M (2015) "Scalable DNA-Based magnetic nanoparticle agglutination assay for bacterial detection in patient samples," ACS Nano, vol. 9, pp. 7374-7382.

Minero G A S, Nogueira C, Rizzi G, Tian B, Fock J, Donolato M, Strömberg M, Hansen M F (2017) "Sequence-specific validation of LAMP amplicons in real-time optomagnetic detection of Dengue serotype 2 synthetic DNA," Analyst, vol. 142, pp. 3441-3450.

Minero G A S, Garbarino F, Fock J, Hansen M F (2018) "Strategies for onchip DNA processing on magnetic microbeads" 12th International Conference on the Scientific and Clinical Applications of Magnetic Carriers, poster 251.

Minero G A S, Cangiano V, Garbarino F, Fock J, Hansen M F (2019) "Integration of microbead DNA handling with optomagnetic detection in RCA assay," Microchim. Acta, vol. 186. pp. 528-538.

Nilsson M, Malmgren H, Samiotaki M, Kwiatkowski M, Chowdhary B P, Landegren U (1994) "Padlock probes: Circularizing oligonucleotides for localized DNA detection," Science, vol. 265, pp. 2085-2088.

Nilsson M, Gullberg M, Dahl F, Szuhai K, Raap A K (2002) "Real-time monitoring of rolling-circle amplification using a modified molecular beacon design," Nucleic Acids Res., vol. 30, pp. e66.

Pashchenko O, Shelby T, Banerjee T, Santra S (2018) "A comparison of optical, electrochemical, magnetic, and colorimetric point-of-care biosensors for infectious disease diagnosis," ACS Infectious Diseases, vol. 4, pp. 1162-1178.

Pavankumar A R, Engström A, Liu J, Herthnek D, Nilsson M (2016) "Proficient Detection of Multi-Drug-Resistant Mycobacterium tuberculosis by Padlock Probes and Lateral Flow Nucleic Acid Biosensors," Anal. Chem., vol. 88, pp. 4277-4284.

Rageade F, Picot N, Blanc-Michaud A, Chatellier S, Mirande C, Fortin E, Belkum A (2014) "Performance of solid and liquid culture media for the detection of Mycobacterium tuberculosis in clinical materials: Meta-analysis of recent studies," European Journal of Clinical Microbiology and Infectious Diseases, vol. 33, pp. 867-870.

Sato K, Tachihara A, Renberg B, Mawatari K, Sato K, Tanaka Y, Jarvius J, Nilsson M, Kitamori T (2010) "Microbead-based rolling circle amplification in a microchip for sensitive DNA detection," Lab Chip, vol. 10, pp. $1262-1266$.

World Health Organization: global tuberculosis report 2018 http://www.who.int/tb/publications/global_report/en/

Zhang S, Wu Z, Shen G, Yu R (2009) "A label-free strategy for SNP detection with high fidelity and sensitivity based on ligation-rolling circle amplification and intercalating of methylene blue," Biosens. Bioelectron., vol. 24, pp. 3201-3207.

Zhou H, Liu J, Xu J J, Zhang S S, and Chen H Y (2018) "Optical nanobiosensing interface: Via nucleic acid amplification strategy: Construction and application," Chemical Society Reviews, vol. 47, pp. 1996-2019.

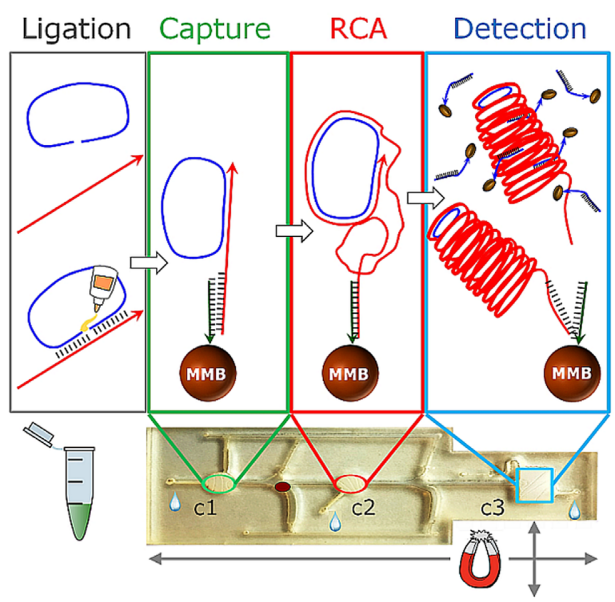

Fig. 1. On-chip integration of RCA with OM detection. (a) Off-chip PLP annealing and ligation was followed by on-chip target capture (c1), RCA (c2), and OM detection (c3). The DNA was transported between reaction chambers $\mathrm{c} 1-\mathrm{c} 2-\mathrm{c} 3$ using $\mathrm{MMBs}$ and an $\mathrm{XZ}$ motorized external magnet. During measurements, the chip was mounted horizontally and sandwiched between two heaters, and a light path through the measurement chamber was formed in the horizontal direction.

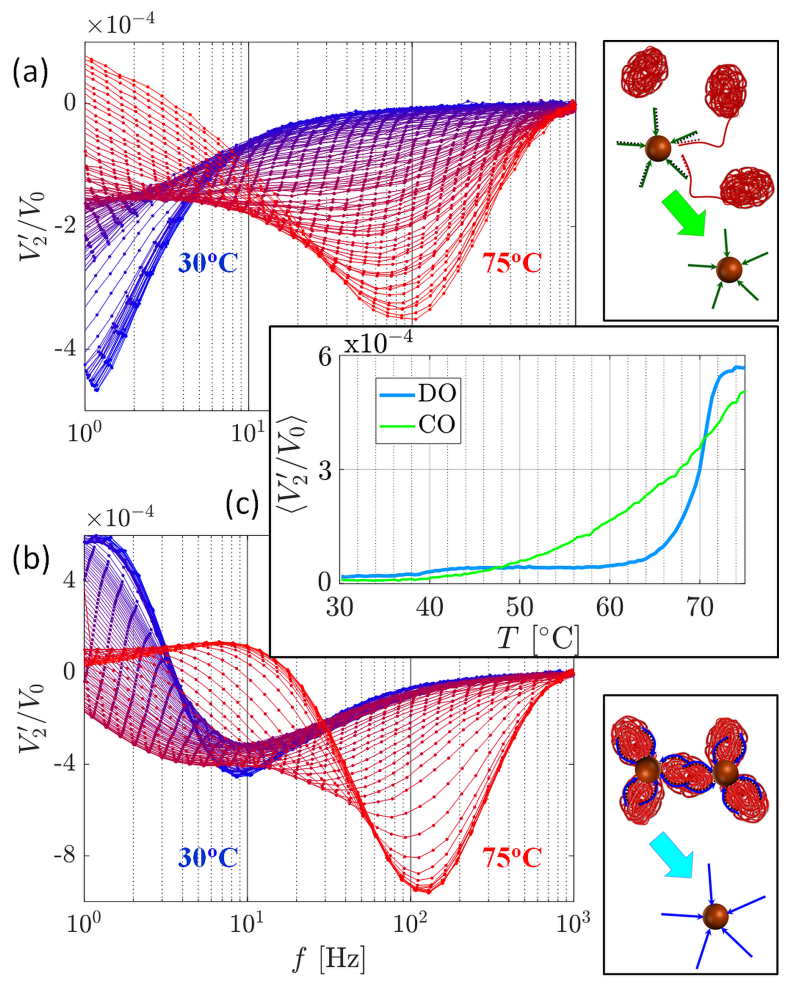

Fig. 2. DNA temperature melting analysis of (a) CO-target and (b) DO-RCP hybrids. In (a) templates for RCA were anchored to MNPs via the CO-target hybrid and RCA was run for $45 \mathrm{~min}$. In (b) readymade RCPs were anchored to DO-MNPs via DO-RCP hybridization. Several DOs as well as several DO-MNPs could bind to each RCP resulting in MNP clusters. OM spectra were recorded after magnetic separation and MNP resuspension in detection buffer followed by stepwise $0.45^{\circ} \mathrm{C}$ heating of the RCP-MNPs between each OM spectrum (45 s). (c) Analysis of the average signal from free MNPs, $\left\langle V_{2}^{\prime} / V_{0}\right\rangle$, vs. temperature for the two series of spectra. 


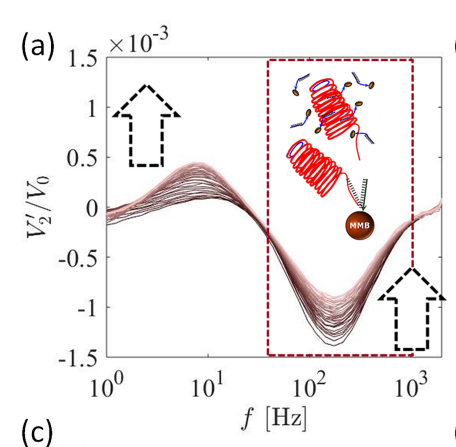

(b)

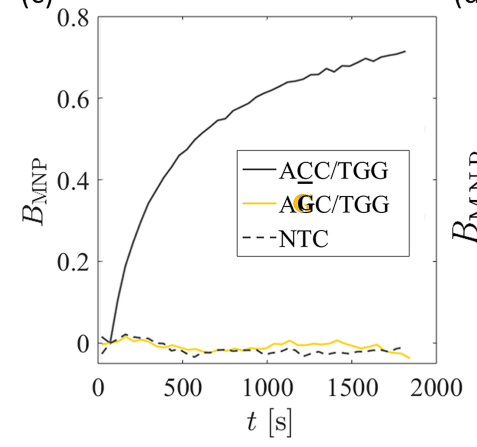

(d) 0.8
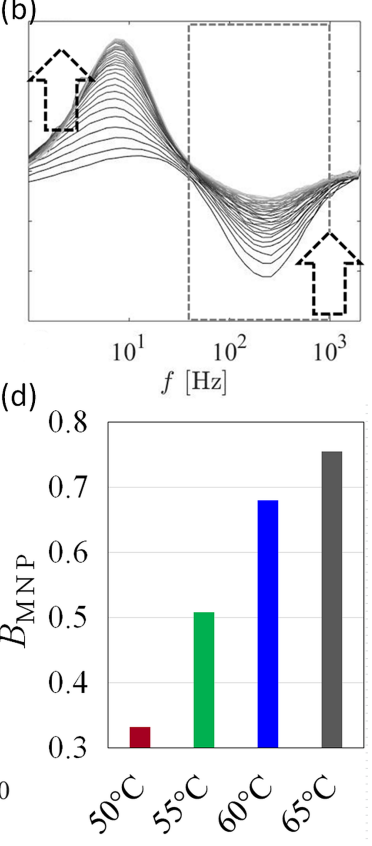

Fig. 3. OM detection of RCPs produced off-chip from $60 \mathrm{pM}$ Mut target with a matching PLP attached to CO-MMBs. After RCA, the MMBs were transferred to a chip and mixed with DO-MNPs. OM spectra were obtained at (a) $50^{\circ} \mathrm{C}$ and (b) $65^{\circ} \mathrm{C}$ vs. time (dark to light color, one spectrum every $45 \mathrm{~s}$ ). The inset shows the proposed strategy of the RCP release from the MMBs through DNA melting at $T \geq 50^{\circ} \mathrm{C}$. The vertical dashed lines indicate the frequency window used to quantify the average signal from free MNPs, $\left\langle V_{2}^{\prime} / V_{0}\right\rangle$. (c) Time evolution of the relative signal from bound MNPs, $B_{\mathrm{MNP}}=1-$ $\left\langle V_{2}^{\prime} / V_{0}\right\rangle(t) /\left\langle V_{2}^{\prime} / V_{0}\right\rangle\left(t_{0}\right)$ during OM detection (45 s per spectrum) at the $62.5^{\circ} \mathrm{C}$ detection temperature. Comparison of assay performance with a Mut PLP for Mut and WT targets and a no target control (NTC). (d) Values of $B_{\mathrm{MNP}}$ after $30 \mathrm{~min}$ of detection at the indicated detection temperatures.
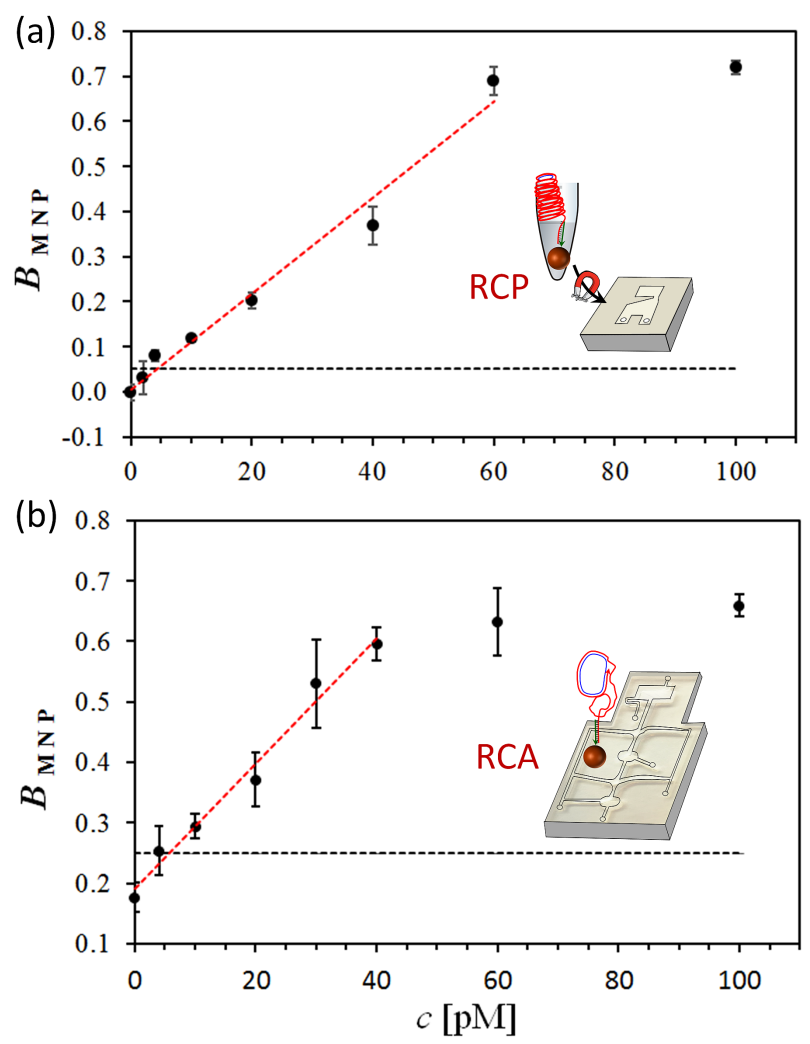

Fig. 4. Dose-response analysis of RCPs synthesized (a) off-chip and (b) on-chip. The OM detection of the fraction $B_{\mathrm{MNP}}$ of MNPs bound to the RCPs after 30 min of monitoring at $62.5^{\circ} \mathrm{C}$. Error bars indicate the standard deviation (SD) for $n=4$. Horizontal lines indicate signal cutoffs (mean $+3 \times$ SD of NTC) and red dashed lines are the linear trends. The LODs were approximated as the crossing points between the cutoffs and the linear trends. 